\title{
Upper Bounds of Schubert Polynomials
}

\author{
Neil J.Y. Fan ${ }^{1}$ and Peter L. Guo ${ }^{2}$ \\ ${ }^{1}$ Department of Mathematics \\ Sichuan University, Chengdu, Sichuan 610064, P.R. China \\ ${ }^{2}$ Center for Combinatorics, LPMC \\ Nankai University, Tianjin 300071, P.R. China \\ ${ }^{1}$ fan@scu.edu.cn, ${ }^{2} \operatorname{lgu}$ @ @nankai.edu.cn
}

\begin{abstract}
Let $w$ be a permutation of $\{1,2, \ldots, n\}$, and let $D(w)$ be the Rothe diagram of $w$. The Schubert polynomial $\mathfrak{S}_{w}(x)$ can be realized as the dual character of the flagged Weyl module associated to $D(w)$. This implies a coefficient-wise inequality

$$
\operatorname{Min}_{w}(x) \leq \mathfrak{S}_{w}(x) \leq \operatorname{Max}_{w}(x),
$$

where both $\operatorname{Min}_{w}(x)$ and $\operatorname{Max}_{w}(x)$ are polynomials determined by $D(w)$. Fink, Mészáros and St. Dizier found that $\mathfrak{S}_{w}(x)$ equals the lower bound $\operatorname{Min}_{w}(x)$ if and only if $w$ avoids twelve permutation patterns. In this paper, we show that $\mathfrak{S}_{w}(x)$ reaches the upper bound $\operatorname{Max}_{w}(x)$ if and only if $w$ avoids two permutation patterns 1432 and 1423. Similarly, for any given composition $\alpha \in \mathbb{Z}_{\geq 0}^{n}$, one can define a lower bound $\operatorname{Min}_{\alpha}(x)$ and an upper bound $\operatorname{Max}_{\alpha}(x)$ for the key polynomial $\kappa_{\alpha}(x)$. Hodges and Yong established that $\kappa_{\alpha}(x)$ equals $\operatorname{Min}_{\alpha}(x)$ if and only if $\alpha$ avoids five composition patterns. We show that $\kappa_{\alpha}(x)$ equals $\operatorname{Max}_{\alpha}(x)$ if and only if $\alpha$ avoids a single composition pattern $(0,2)$. As an application, we obtain that when $\alpha$ avoids $(0,2)$, the key polynomial $\kappa_{\alpha}(x)$ is Lorentzian, partially verifying a conjecture of Huh, Matherne, Mészáros and St. Dizier.
\end{abstract}

\section{Introduction}

The Schubert polynomials $\mathfrak{S}_{w}(x)=\mathfrak{S}_{w}\left(x_{1}, \ldots, x_{n}\right)$ indexed by permutations $w$ of $[n]=$ $\{1,2, \ldots, n\}$ were introduced by Lascoux and Schützenberger [22], representing cohomology classes of Schubert cycles in flag varieties. For combinatorial constructions of Schubert polynomials, see for example [3, 4, 16, 20, 26].

Kraśkiewicz and Pragacz [17,18] proved that $\mathfrak{S}_{w}(x)$ equals the dual character of the flagged Weyl module associated to the Rothe diagram $D(w)$ of $w$. Given a permutation $w=w_{1} w_{2} \cdots w_{n}$ of $[n]$, the Rothe diagram $D(w)=\left(D(w)_{1}, D(w)_{2}, \ldots, D(w)_{n}\right)$ of $w$ is defined by

$$
D(w)_{j}=\left\{i: w_{i}>j, i<w_{j}^{-1}\right\}, \quad \text { where } 1 \leq j \leq n .
$$

In general, a diagram means an ordered list $D=\left(D_{1}, D_{2}, \ldots, D_{n}\right)$ of $n$ subsets of $[n]$. A diagram $D$ can be viewed as a collection of boxes of an $n \times n$ grid, this is, $D_{j}$ consists of 
the boxes $(i, j)$ in row $i$ and column $j$ where $i \in D_{j}$. Here the row indices increase from top to bottom, and the column indices increase from left to right. For example, Figure 1.1(a) represents the diagram $(\{1\},\{4\},\{1,2,4\},\{2\})$. When viewed as a subset of an $n \times n$ grid, the Rothe diagram $D(w)$ can be obtained by removing the boxes that are to the right of $\left(i, w_{i}\right)$ or below $\left(i, w_{i}\right)$. Figure 1.1(b) is the Rothe diagram of 1432 .

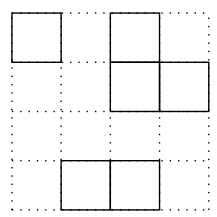

(a)

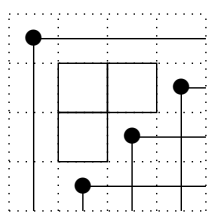

(b)

Figure 1.1: (a) is a diagram, (b) is the Rothe diagram of $w=1432$.

For two diagrams $C=\left(C_{1}, \ldots, C_{n}\right)$ and $D=\left(D_{1}, \ldots, D_{n}\right)$, write $C \leq D$ if $C_{j} \leq D_{j}$ for every $1 \leq j \leq n$, where $C_{j} \leq D_{j}$ means that

(1) $\left|C_{j}\right|=\left|D_{j}\right|$;

(2) for $1 \leq k \leq\left|C_{j}\right|$, the $k$-th least element of $C_{j}$ is less than or equal to the $k$-th least element of $D_{j}$.

It is worth mentioning that the set $\left\{C_{j}: C_{j} \leq D_{j}\right\}$ forms the basis of the Schubert matroid corresponding to $D_{j}$, see for example [1, 5, 9].

Write $x^{D}$ for the monomial generated by a diagram $D$ :

$$
x^{D}=\prod_{j=1}^{n} \prod_{i \in D_{j}} x_{i}
$$

Recall that $D(w)$ is the Rothe diagram of a permutation $w$. Denote

$$
\operatorname{Min}_{w}(x)=\sum_{x^{\beta} \in\left\{x^{C}: C \leq D(w)\right\}} x^{\beta} \quad \text { and } \quad \operatorname{Max}_{w}(x)=\sum_{C \leq D(w)} x^{C} .
$$

Note that the coefficient of each monomial appearing in $\operatorname{Min}_{w}(x)$ equals one.

Given a diagram $D$, one can construct the flagged Weyl module $\mathcal{M}_{D}$ of the group $B$ of invertible upper-triangular $n \times n$ matrices over $\mathbb{C}[10,11,17,18,24]$, see Section 2 for detailed descriptions. Kraśkiewicz and Pragacz [17, 18] showed that $\mathfrak{S}_{w}(x)$ equals the dual character of $\mathcal{M}_{D(w)}$. As a consequence, one has the following coefficient-wise inequality:

$$
\operatorname{Min}_{w}(x) \leq \mathfrak{S}_{w}(x) \leq \operatorname{Max}_{w}(x),
$$

where, for two polynomials $f(x)=\sum_{\beta} a_{\beta} x^{\beta}$ and $g(x)=\sum_{\beta} b_{\beta} x^{\beta}$ in $\mathbb{Z}\left[x_{1}, \ldots, x_{n}\right]$, $f(x) \leq g(x)$ means that $a_{\beta} \leq b_{\beta}$ for any $\beta$. The above inequality (1.1) will also be explained in Section 2 , 
Fink, Mészáros and St. Dizier [11, Theorem 1.1] proved that $\mathfrak{S}_{w}(x)$ attains the lower bound $\operatorname{Min}_{w}(x)$ if and only if $w$ avoids twelve permutation patterns: 12543, 13254, 13524, 13542, 21543, 125364, 125634, 215364, 215634, 315264, 315624, 315642. For a permutation $w$ of $[n]$ and a permutation $\pi$ of $[m]$ with $n \geq m$, we say that $w$ avoids the pattern $\pi$ if there do not exist subsequences in $w$ of length $m$ that are order isomorphic to $\pi$. A Schubert polynomial that reaches the lower bound is called a zero-one Schubert polynomial in [11], which can also be generated exactly by the lattice points in its associated Newton polytope [10].

Our first result provides a characterization of when $\mathfrak{S}_{w}(x)$ reaches the upper bound.

Theorem 1.1. The Schubert polynomial $\mathfrak{S}_{w}(x)$ equals $\operatorname{Max}_{w}(x)$ if and only if $w$ avoids the patterns 1432 and 1423.

Huh, Matherne, Mészáros and St. Dizier [14, Conjecture 15] conjectured that for any permutation $w$, the normalized Schubert polynomial $\mathrm{N}\left(\mathfrak{S}_{w}(x)\right)$ is Lorentzian, where $\mathrm{N}$ is a linear operator defined by

$$
\mathrm{N}\left(x^{\mu}\right)=\frac{x^{\mu}}{\mu !}=\frac{x_{1}^{\mu_{1}} \cdots x_{n}^{\mu_{n}}}{\mu_{1} ! \cdots \mu_{n} !}, \quad \text { for } \mu=\left(\mu_{1}, \ldots, \mu_{n}\right) \in \mathbb{Z}_{\geq 0}^{n} .
$$

We refer the reader to [6] or [14, Definition 5] for several equivalent definitions of Lorentzian polynomials. It should be pointed out that, as an important consequence of the Lorentzian property, the coefficients of a Lorentzian polynomial are log-concave. Using Theorem 1.1 combined with results in [6, 10], Huh, Matherne, Mészáros and St. Dizier [14, Proposition 17] confirmed the above conjecture for permutations avoiding 1432 and 1423. In fact, [14, Proposition 17] proved that when $w$ avoids 1432 and 1423, the Schubert polynomial $\mathfrak{S}_{w}(x)$ is Lorentzian. This is stronger because the Lorentzian property of a polynomial $f(x)$ implies that of $\mathrm{N}(f(x))$ [6, Corollary 3.7]. As noted below [14, Proposition 17], the Schubert polynomials $\mathfrak{S}_{1432}(x)$ and $\mathfrak{S}_{1423}(x)$ are not Lorentzian.

We also remark that Gire [12] showed that the number of permutations of $[n]$ avoiding 2341 and 3241 is the large Schröder number $r_{n-1}$ (see also Kremer [19]), which can be defined via the following generating function

$$
\sum_{r \geq 0} r_{n} x^{n}=\frac{1-x-\sqrt{1-6 x+x^{2}}}{2 x} .
$$

The first few values of $r_{n}$ are 1,2, 6, 22, 90, 394, 1806, 8558, 41586, ... Reversing the order of permutations leads to a bijection between permutations of [ $n$ ] avoiding 1432 and 1423 and permutations of $[n]$ avoiding 2341 and 3241. Thus the number of permutations of $[n]$ avoiding 1432 and 1423 is equal to $r_{n-1}$.

Using analogous arguments, we can characterize when key polynomials reach their upper bounds. Key polynomials $\kappa_{\alpha}(x)$ associated to compositions $\alpha \in \mathbb{Z}_{\geq 0}^{n}$ (also called Demazure characters) are characters of the Demazure modules for the general linear groups [7, 8, 10]. Their combinatorial properties were initially investigated by Lascoux and Schützenberger [21]. It is known that every Schubert polynomial is a positive sum 
of key polynomials [23,25]. It is also worth mentioning that $\kappa_{\alpha}(x)$ can be realized as a specialization of the nonsymmetric Macdonald polynomial $E_{\alpha}(x ; q, t)$ at $q=t=0[2,15$.

The key polynomial $\kappa_{\alpha}(x)$ is equal to the dual character of the flagged Weyl module $\mathcal{M}_{D(\alpha)}$ associated to the skyline diagram $D(\alpha)$ of $\alpha$ [7, 8, 10]. Recall that the skyline diagram $D(\alpha)$ consists of the first $\alpha_{i}$ boxes in row $i$. For example, Figure 1.2 depicts the skyline diagram of $(1,3,0,2)$.

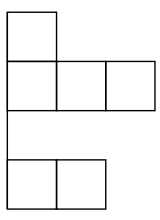

Figure 1.2: The skyline diagram of $(1,3,0,2)$.

Write

$$
\operatorname{Min}_{\alpha}(x)=\sum_{x^{\beta} \in\left\{x^{C}: C \leq D(\alpha)\right\}} x^{\beta} \quad \text { and } \quad \operatorname{Max}_{\alpha}(x)=\sum_{C \leq D(\alpha)} x^{C} .
$$

Similar to Schubert polynomials, one has the following inequality:

$$
\operatorname{Min}_{\alpha}(x) \leq \kappa_{\alpha}(x) \leq \operatorname{Max}_{\alpha}(x) .
$$

Recently, Hodges and Yong [13] established a pattern avoidance characterization of when $\kappa_{\alpha}(x)$ equals the lower bound $\operatorname{Min}_{\alpha}(x)$, which they call multiplicity-free key polynomials. For two compositions $\alpha=\left(\alpha_{1}, \ldots, \alpha_{n}\right)$ and $\beta=\left(\beta_{1}, \ldots, \beta_{m}\right)$ with $n \geq m$, we say that $\alpha$ contains the composition pattern $\beta$ if there exists $i_{1}<i_{2}<\cdots<i_{m}$ such that

(1) $\alpha_{i_{s}} \leq \alpha_{i_{t}}$ if and only if $\beta_{s} \leq \beta_{t}$;

(2) $\left|\alpha_{i_{s}}-\alpha_{i_{t}}\right| \geq\left|\beta_{s}-\beta_{t}\right|$.

We say that $\alpha$ avoids $\beta$ if $\alpha$ does not contain the pattern $\beta$. Using the quasi-key model along with the Kohnert diagram model of key polynomials, Hodges and Yong [13] showed that $\kappa_{\alpha}(x)$ equals $\operatorname{Min}_{\alpha}(x)$ if and only if $\alpha$ avoids the following five composition patterns:

$$
(0,1,2),(0,0,2,2),(0,0,2,1),(1,0,3,2),(1,0,2,2) .
$$

Our second result gives a characterization of when $\kappa_{\alpha}(x)$ reaches the upper bound.

Theorem 1.2. The key polynomial $\kappa_{\alpha}(x)$ equals $\operatorname{Max}_{\alpha}(x)$ if and only if $\alpha$ avoids the composition pattern $(0,2)$, that $i s$, there do not exist $i<j$ such that $\alpha_{j}-\alpha_{i} \geq 2$.

Huh, Matherne, Mészáros and St. Dizier [14, Conjecture 23] conjectured that for any composition $\alpha \in \mathbb{Z}_{\geq 0}^{n}$, the normalized key polynomial $\mathrm{N}\left(\kappa_{\alpha}(x)\right)$ is Lorentzian. Notice that the support of $\kappa_{\alpha}(x)$ is the set of integral points in the Minkowski sum of matroid polytopes associated to the skyline diagram $D(\alpha)$ [10, Theorem 11]. This, along with Theorem 1.2, enables us to invoke the same arguments as in the proof of [14, Proposition 17 to verify the above conjecture for compositions avoiding $(0,2)$. 
Corollary 1.3. If $\alpha$ avoids the composition pattern $(0,2)$, then the key polynomial $\kappa_{\alpha}(x)$ is Lorentzian, and hence $\mathrm{N}\left(\kappa_{\alpha}(x)\right)$ is Lorentzian.

Note that the key polynomial $\kappa_{(0,2)}(x)$ is not Lorentzian. This is because $\kappa_{(0,2)}(x)$ equals the Schubert polynomial $\mathfrak{S}_{1423}(x)$, and the latter is not Lorentzian, as mentioned after Theorem 1.1.

This paper is structured as follows. In Section 2, we give an overview of flagged Weyl modules as well as the fact that Schubert and key polynomials are the dual characters of flagged Weyl modules respectively associated to Rothe diagrams and skyline diagrams. We complete the proofs of Theorems 1.1 and 1.2 respectively in Sections 3 and 4 .

\section{Dual characters of flagged Weyl modules}

Let us start with an overview of the flagged Weyl module $\mathcal{M}_{D}$ associated to a diagram $D$. The module $\mathcal{M}_{D}$ can be constructed by means of determinants [24]. Here we use the notation in $[10,11$. Let $\operatorname{GL}(n, \mathbb{C})$ be the group of $n \times n$ invertible matrices over $\mathbb{C}$, and let $B$ be the subgroup consisting of the $n \times n$ upper-triangular matrices. Let $Y$ be the $n \times n$ upper-triangular matrix whose entries are indeterminates $y_{i j}$ where $i \leq j$. Denote by $\mathbb{C}[Y]$ the ring of polynomials in the variables $\left\{y_{i j}\right\}_{i \leq j}$. The group $\operatorname{GL}(n, \mathbb{C})$ acts on $\mathbb{C}[Y]$ (on the right) as follows. Given a matrix $g \in \operatorname{GL}(n, \mathbb{C})$ and a polynomial $f(Y) \in \mathbb{C}[Y]$,

$$
f(Y) \cdot g=f\left(g^{-1} Y\right) .
$$

To a diagram $D=\left(D_{1}, \ldots, D_{n}\right)$, the associated flagged Weyl module $\mathcal{M}_{D}$ is a $B$-module defined by

$$
\mathcal{M}_{D}=\operatorname{Span}_{\mathbb{C}}\left\{\prod_{j=1}^{n} \operatorname{det}\left(Y_{D_{j}}^{C_{j}}\right): C \leq D\right\},
$$

where, for two subsets $R$ and $S$ of $[n], Y_{S}^{R}$ denotes the submatrix of $Y$ with row indices

in $R$ and column indices in $S$. It should be noted that $\prod_{j=1}^{n} \operatorname{det}\left(Y_{D_{j}}^{C_{j}}\right) \neq 0$ if and only if $C \leq D$.

Let $X=\operatorname{diag}\left(x_{1}, \ldots, x_{n}\right)$ be a diagonal matrix, which can be viewed as a linear transformation from $\mathcal{M}_{D}$ to $\mathcal{M}_{D}$ via the $B$-action. The character of $\mathcal{M}_{D}$ is defined as the trace of $X$ :

$$
\operatorname{char}\left(\mathcal{M}_{D}\right)(x)=\operatorname{tr}\left(X: \mathcal{M}_{D} \rightarrow \mathcal{M}_{D}\right) .
$$

The dual character of $\mathcal{M}_{D}$ is the character of the dual module $\mathcal{M}_{D}^{*}$, which is given by

$$
\begin{aligned}
\operatorname{char}^{*}\left(\mathcal{M}_{D}\right)(x) & =\operatorname{tr}\left(X: \mathcal{M}_{D}^{*} \rightarrow \mathcal{M}_{D}^{*}\right) \\
& =\operatorname{char}\left(\mathcal{M}_{D}\right)\left(x_{1}^{-1}, \ldots, x_{n}^{-1}\right)
\end{aligned}
$$

The Schubert and key polynomials are equal to the dual characters of flag Weyl modules respectively corresponding to the Rothe and skyline diagrams. Recall that 
Schubert polynomials are defined based on the divided difference operator $\partial_{i}$, which sends a polynomial $f(x) \in \mathbb{Z}\left[x_{1}, \ldots, x_{n}\right]$ to

$$
\partial_{i} f(x)=\frac{f(x)-s_{i} f(x)}{x_{i}-x_{i+1}}
$$

where $s_{i} f(x)$ is obtained from $f(x)$ by exchanging $x_{i}$ and $x_{i+1}$. For the longest permutation $w_{0}=n(n-1) \cdots 1$, set $\mathfrak{S}_{w_{0}}(x)=x_{1}^{n-1} x_{2}^{n-2} \cdots x_{n-1}$. For $w \neq w_{0}$, there exists a position $1 \leq i<n$ such that $w_{i}<w_{i+1}$. Let $w s_{i}$ be the permutation obtained from $w$ by interchanging $w_{i}$ and $w_{i+1}$. Set $\mathfrak{S}_{w}(x)=\partial_{i} \mathfrak{S}_{w s_{i}}(x)$. The above definition is independent of the choice of $i$ since the operators $\partial_{i}$ satisfy the braid relations: $\partial_{i} \partial_{j}=\partial_{j} \partial_{i}$ for $|i-j|>1$, and $\partial_{i} \partial_{i+1} \partial_{i}=\partial_{i+1} \partial_{i} \partial_{i+1}$.

As mentioned above, $\mathfrak{S}_{w}(x)$ coincides with the dual character of $\mathcal{M}_{D(w)}$ [17, 18]:

$$
\mathfrak{S}_{w}(x)=\operatorname{char}^{*}\left(\mathcal{M}_{D(w)}\right)(x) .
$$

For $C \leq D$, the effect of the action of $X$ on the polynomial $\prod_{j=1}^{n} \operatorname{det}\left(Y_{D_{j}}^{C_{j}}\right)$ is

$$
\prod_{j=1}^{n} \operatorname{det}\left(Y_{D_{j}}^{C_{j}}\right) \cdot X=\prod_{j=1}^{n} \prod_{i \in C_{j}} x_{i}^{-1} \cdot \prod_{j=1}^{n} \operatorname{det}\left(Y_{D_{j}}^{C_{j}}\right) .
$$

Thus the polynomial $\prod_{j=1}^{n} \operatorname{det}\left(Y_{D_{j}}^{C_{j}}\right)$ is an eigenvector of $X$ with eigenvalue

$$
\prod_{j=1}^{n} \prod_{i \in C_{j}} x_{i}^{-1}
$$

Therefore, the set of monomials appearing in $\mathfrak{S}_{w}(x)$ is exactly

$$
\left\{x^{C}: C \leq D(w)\right\} \text {. }
$$

Moreover, the coefficient of a monomial $x^{\alpha}$ appearing in $\mathfrak{S}_{w}(x)$ is equal to the dimension of the corresponding eigenspace

$$
\operatorname{Span}_{\mathbb{C}}\left\{\prod_{j=1}^{n} \operatorname{det}\left(Y_{D_{j}}^{C_{j}}\right): C \leq D(w), x^{C}=x^{\alpha}\right\} .
$$

By the above observations, we obtain the lower and the upper bounds for Schubert polynomials as given in (1.1).

Obviously, $\mathfrak{S}_{w}(x)$ equals the lower bound $\operatorname{Min}_{w}(x)$ if and only if for each monomial $x^{\alpha}$ appearing in $\mathfrak{S}_{w}(x)$, the eigenspace in (2.3) has dimension one. While, $\mathfrak{S}_{w}(x)$ equals the upper bound $\operatorname{Max}_{w}(x)$ if and only if for each monomial $x^{\alpha}$ appearing in $\mathfrak{S}_{w}(x)$, the dimension of the eigenspace in (2.3) is

$$
\#\left\{C: C \leq D(w), x^{C}=x^{\alpha}\right\},
$$

that is, the collection of polynomials

$$
\prod_{j=1}^{n} \operatorname{det}\left(Y_{D_{j}}^{C_{j}}\right), \quad \text { where } C \leq D(w)
$$


are linearly independent.

Let us use an example to illustrate (2.2). Consider the permutation $w=1432$. We have $D(1432)=(\emptyset,\{2,3\},\{2\}, \emptyset)$. There are six diagrams $C \leq D(1432)$ as listed below:

$$
\begin{aligned}
& C^{(1)}=(\emptyset,\{1,2\},\{1\}, \emptyset), \quad C^{(2)}=(\emptyset,\{1,3\},\{1\}, \emptyset), \quad C^{(3)}=(\emptyset,\{2,3\},\{1\}, \emptyset), \\
& C^{(4)}=(\emptyset,\{1,2\},\{2\}, \emptyset), \quad C^{(5)}=(\emptyset,\{1,3\},\{2\}, \emptyset), \quad C^{(6)}=(\emptyset,\{2,3\},\{2\}, \emptyset) .
\end{aligned}
$$

Notice that the diagrams $C^{(3)}$ and $C^{(5)}$ give rise to the same monomial $y_{12} y_{22} y_{33}$. So the module $\mathcal{M}_{D(w)}$ is spanned by the following set of polynomials

$$
\left\{\left(y_{12} y_{23}-y_{13} y_{23}\right) y_{12},\left(y_{12} y_{23}-y_{13} y_{23}\right) y_{22}, y_{12}^{2} y_{33}, y_{12} y_{22} y_{33}, y_{22}^{2} y_{33}\right\} \text {. }
$$

It is easily checked that the above five polynomials are linearly independent. So,

$$
\begin{aligned}
\operatorname{char}^{*}\left(\mathcal{M}_{D(1432)}\right)(x) & =x^{C^{(1)}}+x^{C^{(2)}}+x^{C^{(3)}}+x^{C^{(4)}}+x^{C^{(6)}} \\
& =x_{1}^{2} x_{2}+x_{1}^{2} x_{3}+x_{1} x_{2} x_{3}+x_{1} x_{2}^{2}+x_{2}^{2} x_{3},
\end{aligned}
$$

which agrees with the Schubert polynomial $\mathfrak{S}_{1432}(x)$.

We finally turn to key polynomials. Key polynomials are defined using the Demazure operator $\pi_{i}=\partial_{i} x_{i}$. If $\alpha$ is a partition, set $\kappa_{\alpha}(x)=x^{\alpha}$. Otherwise, choose $i$ such that $\alpha_{i}<\alpha_{i+1}$. Let $\alpha^{\prime}$ be the composition obtained from $\alpha$ by interchanging $\alpha_{i}$ and $\alpha_{i+1}$. Set $\kappa_{\alpha}(x)=\pi_{i} \kappa_{\alpha^{\prime}}(x)$. The key polynomial $\kappa_{\alpha}(x)$ equals the dual character of the flag Weyl module associated to the skyline diagram $D(\alpha)$ :

$$
\kappa_{\alpha}(x)=\operatorname{char}^{*}\left(\mathcal{M}_{D(\alpha)}\right)(x) .
$$

In view of the arguments for Schubert polynomials, we obtain the lower and the upper bounds for key polynomials given in (1.2).

\section{Proof of Theorem 1.1}

In this section, we shall prove the necessity and the sufficiency of Theorem 1.1 in Theorem 3.1 and Theorem 3.2 , respectively.

Theorem 3.1. If $\mathfrak{S}_{w}(x)=\operatorname{Max}_{w}(x)$, then $w$ avoids the patterns 1432 and 1423 .

Proof. We first show that if $\mathfrak{S}_{w}(x)=\operatorname{Max}_{w}(x)$, then $w$ must avoid 1432. The proof is by contradiction. Suppose otherwise that $w$ contains a subsequence that is order isomorphic to 1432 . Let $i_{0}$ be the largest $i$ such that $w_{i} w_{k} w_{p} w_{q}$ is order isomorphic to 1432 . Once $i_{0}$ is determined, let $k_{0}$ be the smallest $k$ such that $w_{i_{0}} w_{k_{0}} w_{p} w_{q}$ is order isomorphic to 1432. Now, let $w_{i_{0}} w_{k_{0}} w_{p_{0}} w_{q_{0}}$ be any fixed subsequence order isomorphic to 1432. By the choices of $i_{0}$ and $k_{0}$, we see that $w_{q_{0}}<w_{i}<w_{p_{0}}$ for any $i_{0}<i<k_{0}$. Denote $j_{0}=w_{q_{0}}$ and $l_{0}=w_{p_{0}}$. We have the following two observations.

O 1. The box $\left(i_{0}, j_{0}\right) \notin D(w)$. For $i_{0}<i \leq k_{0}$, the box $\left(i, j_{0}\right) \in D(w)$. 


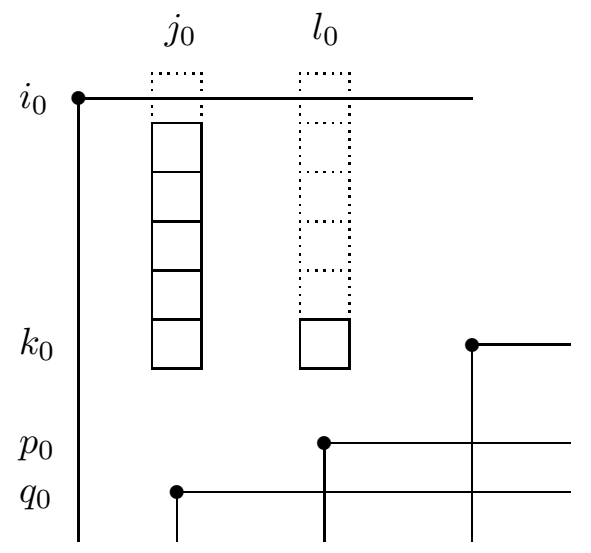

Figure 3.3: Local configuration of the boxes in column $j_{0}$ and column $l_{0}$.

O 2. The box $\left(k_{0}, l_{0}\right) \in D(w)$. For $i_{0} \leq i<k_{0}$, the box $\left(i, l_{0}\right) \notin D(w)$.

So the configuration of the boxes of $D(w)$ in column $j_{0}$ and column $l_{0}$ that lie between row $i_{0}$ and row $k_{0}$ is as illustrated in Figure 3.3.

Assume that $D(w)=\left(D_{1}, \ldots, D_{n}\right)$. For ease of description, we denote the polynomial generated by a diagram $C \leq D(w)$ by

$$
f_{C}(Y)=\prod_{j=1}^{n} \operatorname{det}\left(Y_{D_{j}}^{C_{j}}\right)
$$

Let $t=k_{0}-i_{0}+1$. We shall construct $t$ distinct diagrams $C^{(1)}, \ldots, C^{(t)}$ such that the corresponding polynomials $f_{C^{(1)}}(Y), \ldots, f_{C^{(t)}}(Y)$ are linearly dependent. For $1 \leq m \leq t$, assume that $C^{(m)}=\left(C_{1}^{(m)}, \ldots, C_{n}^{(m)}\right)$. The diagram $C^{(m)}$ is defined as follows.

(1) For $j \notin\left\{j_{0}, l_{0}\right\}$, let $C_{j}^{(m)}=D_{j}$.

(2) For $j=l_{0}$, let

$$
C_{l_{0}}^{(m)}=\left(D_{l_{0}} \backslash\left\{k_{0}\right\}\right) \cup\left\{i_{0}+m-1\right\} .
$$

(3) For $j=j_{0}$, let

$$
C_{j_{0}}^{(m)}=\left(D_{j_{0}} \cup\left\{i_{0}\right\}\right) \backslash\left\{i_{0}+m-1\right\}
$$

By the above constructions, it is easily seen that $C_{l_{0}}^{(m)} \leq D_{l_{0}}$ and $C_{j_{0}}^{(m)} \leq D_{j_{0}}$, and hence $C^{(m)} \leq D(w)$ for each $1 \leq m \leq t$.

By definition, we have

$$
f_{C^{(m)}}(x)=\operatorname{det}\left(Y_{D_{j_{0}}}^{C_{j_{0}}^{(m)}}\right) \cdot \operatorname{det}\left(Y_{D_{l_{0}}}^{C_{l_{0}}^{(m)}}\right) \cdot \prod_{j \notin\left\{j_{0}, l_{0}\right\}} \operatorname{det}\left(Y_{D_{j}}^{C_{j}^{(m)}}\right) .
$$


Now we evaluate the three factors appearing in (3.2). For $j \notin\left\{j_{0}, l_{0}\right\}$, since $C_{j}^{(m)}=D_{j}$, it follows that $\operatorname{det}\left(Y_{D_{j}}^{C_{j}^{(m)}}\right)$ is an upper-triangular matrix, and thus

$$
\operatorname{det}\left(Y_{D_{j}}^{C_{j}^{(m)}}\right)=\prod_{j \notin\left\{j_{0}, l_{0}\right\}} \prod_{i \in D_{j}} y_{i i} .
$$

To calculate $\operatorname{det}\left(Y_{D_{j_{0}}}^{C_{j_{0}}^{(m)}}\right)$, let

$$
R^{(m)}=\left[i_{0}, k_{0}\right] \backslash\left\{i_{0}+m-1\right\}
$$

and

$$
S=D_{j_{0}} \backslash\left[i_{0}+1, k_{0}\right],
$$

where, for two integers $a<b$, we use $[a, b]$ to denote the interval $\{a, a+1, \ldots, b\}$. Clearly, $C_{j_{0}}^{(m)}$ is the disjoint union of $R^{(m)}$ and $S$, and $D_{j_{0}}$ is the disjoint union of $\left[i_{0}+1, k_{0}\right]$ and $S$. Noticing that the submatrix of $Y_{D_{j_{0}}}^{C_{j_{0}}^{(m)}}$ obtained by restricting both the row and the column indices to $S$ is upper-triangular, we have

$$
\operatorname{det}\left(Y_{D_{j_{0}}}^{C_{j_{0}}^{(m)}}\right)=\operatorname{det}\left(Y_{\left[i_{0}+1, k_{0}\right]}^{R^{(m)}}\right) \cdot \prod_{i \in S} y_{i i} .
$$

Moreover, by the choice of $C_{l_{0}}^{(m)}$, it is easy to see that the matrix $Y_{D_{l_{0}}}^{C_{l_{0}}^{(m)}}$ is an uppertriangular matrix. So we obtain that

$$
\operatorname{det}\left(Y_{D_{l_{0}}}^{C_{l_{0}}^{(m)}}\right)=y_{a_{m} k_{0}} \cdot \prod_{i \in D_{l_{0}} \backslash\left\{k_{0}\right\}} y_{i i}
$$

where $a_{m}=i_{0}+m-1$.

In view of (3.3), (3.4) and (3.5), we find that the polynomials $f_{C^{(1)}}(Y), \ldots, f_{C^{(t)}}(Y)$ have the following common factor

$$
\prod_{j \notin\left\{j_{0}, l_{0}\right\}} \prod_{i \in D_{j}} y_{i i} \cdot \prod_{i \in S} y_{i i} \cdot \prod_{i \in D_{l_{0}} \backslash\left\{k_{0}\right\}} y_{i i}
$$

Therefore, to prove that the polynomials $f_{C^{(1)}}(Y), \ldots, f_{C^{(t)}}(Y)$ are linearly dependent, it is enough to verify that for $1 \leq m \leq t$, the polynomials

$$
g_{m}(Y)=y_{a_{m} k_{0}} \cdot \operatorname{det}\left(Y_{\left[i_{0}+1, k_{0}\right]}^{R^{(m)}}\right)
$$

are linearly dependent. For simplicity, let $b=k_{0}$. Without loss of generality, assume that $i_{0}=1$. Then we have $a_{m}=m$ and $R^{(m)}=[b] \backslash\{m\}$, and hence

$$
g_{m}(Y)=y_{m b} \cdot \operatorname{det}\left(Y_{[2, b]}^{[b] \backslash\{m\}}\right), \quad \text { where } 1 \leq m \leq b .
$$


We claim that

$$
g_{b}(Y)=g_{b-1}(Y)-g_{b-2}(Y)+\cdots+(-1)^{b} g_{1}(Y)
$$

To prove the claim, let us first consider

$$
g_{b}(Y)=y_{b b} \cdot \operatorname{det}\left(Y_{[2, b]}^{[b-1]}\right)
$$

Notice that in the last row of the matrix $Y_{[2, b]}^{[b-1]}$, the only nonzero entries are $y_{(b-1)(b-1)}$ and $y_{(b-1) b}$. Using the Laplace expansion along the last row, we have

$$
g_{b}(Y)=y_{b b}\left(-y_{(b-1)(b-1)} \cdot \operatorname{det}\left(Y_{[2, b] \backslash\{b-1\}}^{[b-2]}\right)+y_{(b-1) b} \cdot \operatorname{det}\left(Y_{[2, b-1]}^{[b-2]}\right)\right) .
$$

Then we consider

$$
g_{b-1}(Y)=y_{(b-1) b} \cdot \operatorname{det}\left(Y_{[2, b]}^{[b] \backslash\{b-1\}}\right) .
$$

Since the last row of $Y_{[2, b]}^{[b] \backslash\{b-1\}}$ has only one nonzero element $y_{b b}$, applying the Laplace expansion along the last row gives

$$
g_{b-1}(Y)=y_{(b-1) b} \cdot y_{b b} \cdot \operatorname{det}\left(Y_{[2, b-1]}^{[b-2]}\right) .
$$

Combining (3.7) and (3.8), we are led to

$$
g_{b}(Y)=g_{b-1}(Y)-y_{b b} \cdot y_{(b-1)(b-1)} \cdot \operatorname{det}\left(Y_{[2, b] \backslash\{b-1\}}^{[b-2]}\right) .
$$

Let us proceed to consider the summand

$$
y_{b b} \cdot y_{(b-1)(b-1)} \cdot \operatorname{det}\left(Y_{[2, b] \backslash\{b-1\}}^{[b-2]}\right)
$$

appearing in (3.9) $)$. Again, applying the Laplace expansion to $Y_{[2, b] \backslash\{b-1\}}^{[b-2]}$ along the last row yields

$$
\begin{aligned}
y_{b b} & \cdot y_{(b-1)(b-1)} \cdot \operatorname{det}\left(Y_{[2, b] \backslash\{b-1\}}^{[b-2]}\right) \\
& =y_{b b} \cdot y_{(b-1)(b-1)}\left(-y_{(b-2)(b-2)} \cdot \operatorname{det}\left(Y_{[2, b] \backslash\{b-1, b-2\}}^{[b-3]}\right)+y_{(b-2) b} \operatorname{det}\left(Y_{[2, b-2]}^{[b-3]}\right)\right) .
\end{aligned}
$$

On the other hand,

$$
\begin{aligned}
g_{b-2}(Y) & =y_{(b-2) b} \cdot \operatorname{det}\left(Y_{[2, b]}^{[b] \backslash\{b-2\}}\right) \\
& =y_{(b-2) b} \cdot y_{b b} \cdot \operatorname{det}\left(Y_{[2, b-1]}^{[b-1] \backslash\{b-2\}}\right) \\
& =y_{(b-2) b} \cdot y_{b b} \cdot y_{(b-1)(b-1)} \cdot \operatorname{det}\left(Y_{[2, b-2]}^{[b-3]}\right) .
\end{aligned}
$$

In view of (3.9), (3.10) and (3.11), it follows that

$$
g_{b}(Y)=g_{b-1}(Y)-g_{b-2}(Y)+y_{b b} \cdot y_{(b-1)(b-1)} \cdot y_{(b-2)(b-2)} \cdot \operatorname{det}\left(Y_{[2, b] \backslash\{b-1, b-2\}}^{[b-3]}\right) .
$$


Continuing the same procedure, we can arrive at the assertion in (3.6) eventually. This implies that the polynomials $g_{m}(Y)$ are linearly dependent, and so the polynomials $f_{C^{(1)}}(Y), \ldots, f_{C^{(t)}}(Y)$ are linearly dependent. Hence we conclude that $\mathfrak{S}_{w}(x) \neq \operatorname{Max}_{w}(x)$.

The same arguments can be employed to show that if $w$ contains a subsequence order-isomorphic to 1423 , then $\mathfrak{S}_{w}(x) \neq \operatorname{Max}_{w}(x)$. In fact, the proof for the case 1432 does not use the relative order of $w_{p_{0}}$ and $w_{q_{0}}$ in the subsequence $w_{i_{0}} w_{k_{0}} w_{p_{0}} w_{q_{0}}$. This completes the proof.

We now prove the sufficiency of Theorem 1.1 .

Theorem 3.2. If $w$ avoids the patterns 1432 and 1423, then $\mathfrak{S}_{w}(x)=\operatorname{Max}_{w}(x)$.

Proof. Suppose that $\{C: C \leq D(w)\}=\left\{C^{(1)}, \ldots, C^{(t)}\right\}$. For a diagram $C$, the polynomial $f_{C}(Y)$ is as defined in (3.1). To prove $\mathfrak{S}_{w}(x)=\operatorname{Max}_{w}(x)$, it is equivalent to show that the polynomials $f_{C^{(1)}}(Y), \ldots, f_{C^{(t)}}(Y)$ are linearly independent.

Assume that $D(w)=\left(D_{1}, \ldots, D_{n}\right)$. For $1 \leq j \leq n$, let

$$
D_{j}^{\prime}=\left\{i \in D_{j} \text { : there exists some } 1 \leq k<i \text { such that } k \notin D_{j}\right\} .
$$

Equivalently, if we let $a_{j}$ be the largest integer such that $\left[a_{j}\right] \subseteq D_{j}$, then

$$
D_{j}^{\prime}=D_{j} \backslash\left[a_{j}\right] .
$$

It is easy to see that for any diagram $C=\left(C_{1}, \ldots, C_{n}\right) \leq D(w)$, we must have $\left[a_{j}\right] \subseteq C_{j}$. This allows us to obtain the following equality

$$
\operatorname{det}\left(Y_{D_{j}}^{C_{j}}\right)=\prod_{i=1}^{a_{j}} y_{i i} \cdot \operatorname{det}\left(Y_{D_{j} \backslash\left[a_{j}\right]}^{C_{j} \backslash\left[a_{j}\right]}\right)=\prod_{i=1}^{a_{j}} y_{i i} \cdot \operatorname{det}\left(Y_{D_{j}^{\prime}}^{C_{j} \backslash\left[a_{j}\right]}\right),
$$

and so we have

$$
f_{C}(Y)=\prod_{j=1}^{n} \prod_{i=1}^{a_{j}} y_{i i} \cdot \prod_{j=1}^{n} \operatorname{det}\left(Y_{D_{j}^{\prime}}^{C_{j} \backslash\left[a_{j}\right]}\right) .
$$

Hence, to show that the polynomials $f_{C^{(1)}}(Y), \ldots, f_{C^{(t)}}(Y)$ are linearly independent, it suffices to show that the following polynomials are linearly independent:

$$
h_{m}(Y)=\prod_{j=1}^{n} \operatorname{det}\left(Y_{D_{j}^{\prime}}^{C_{j}^{(m)} \backslash\left[a_{j}\right]}\right), \quad \text { where } 1 \leq m \leq t .
$$

To this end, we claim that for $1 \leq j_{1}<j_{2} \leq n, D_{j_{1}}^{\prime} \cap D_{j_{2}}^{\prime}=\emptyset$. Suppose otherwise that $D_{j_{1}}^{\prime} \cap D_{j_{2}}^{\prime} \neq \emptyset$. Choose an element $i_{0} \in D_{j_{1}} \cap D_{j_{2}}$. By the definition of $D_{j}^{\prime}$, there exists $1 \leq k<i_{0}$ that does not belong to $D_{j_{1}}$. This means that the box $\left(k, j_{1}\right) \notin D(w)$, implying that $w_{k}<j_{1}$. Now we consider the subsequence $w_{k} w_{i_{0}} w_{p} w_{q}$, where $\left\{w_{p}, w_{q}\right\}=$ $\left\{j_{1}, j_{2}\right\}$. Since the box $\left(i_{0}, j_{2}\right)$ belongs to $D(w)$, it follows that $w_{i_{0}}>j_{2}$. So we have $w_{k}<j_{1}<j_{2}<w_{i_{0}}$. This implies that the subsequence $w_{k} w_{i_{0}} w_{p} w_{q}$ is order isomorphic to 1432 or 1423 , leading to a contradiction. This verifies the claim.

By the above claim, we see that for $1 \leq t_{1} \neq t_{2} \leq t, h_{t_{1}}(Y)$ and $h_{t_{2}}(Y)$ do not contain any monomial in common, which obviously implies that the polynomials $h_{m}(Y)$ are linearly independent. This completes the proof. 


\section{Proof of Theorem 1.2}

The proof of Theorem 1.2 can be carried out in the same vein as Theorem 1.1.

Proof of Theorem 1.2. We first prove the necessity, that is, if $\kappa_{\alpha}(x)=\operatorname{Max}_{\alpha}(x)$, then $\alpha$ avoids the pattern $(0,2)$. Suppose otherwise that $\alpha$ contains a $(0,2)$ pattern. Write $\alpha=\left(\alpha_{1}, \ldots, \alpha_{n}\right)$. Among the $(0,2)$ patterns of $\alpha$, choose the largest index $i_{0}$ such that $\left(\alpha_{i_{0}}, \alpha_{k}\right)$ is a $(0,2)$ pattern for some $k>i_{0}$. Once $i_{0}$ is fixed, locate the smallest index $k_{0}$ such that $\left(\alpha_{i_{0}}, \alpha_{k_{0}}\right)$ is a $(0,2)$ pattern. Since $\left(\alpha_{i_{0}}, \alpha_{k_{0}}\right)$ is a $(0,2)$ pattern, by definition we have

$$
\alpha_{k_{0}}-\alpha_{i_{0}} \geq 2
$$

By the choices of $i_{0}$ and $k_{0}$, it is easy to check that for $i_{0}<i<k_{0}$

$$
\alpha_{i}-\alpha_{i_{0}}=1
$$

By (4.1) and (4.2), the configuration of the boxes of $D(\alpha)$ lying between row $i_{0}$ and row $k_{0}$ is depicted in Figure 4.4. Let $j_{0}=\alpha_{i_{0}}+1$ and $l_{0}=\alpha_{k_{0}}$. Clearly, the configuration

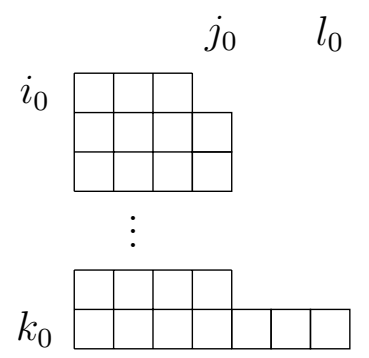

Figure 4.4: An illustration of the boxes of $D(\alpha)$ in row $i_{0}$ and $k_{0}$.

of the boxes of $D(\alpha)$ in column $j_{0}$ and column $l_{0}$ that lie between row $i_{0}$ and row $k_{0}$ is completely that same as that in Figure 3.3. Therefore, using the same arguments as in the proof of Theorem 3.1, we obtain that the assumption that $\alpha$ contains a $(0,2)$ pattern is false. This verifies the necessity.

It remains to prove the sufficiency. The analysis is similar to that in the proof of Theorem 3.2, and is sketched below. Assume that $\alpha$ avoids the composition pattern $(0,2)$. Write $D(\alpha)=\left(D_{1}, \ldots, D_{n}\right)$. For $1 \leq j \leq n$, let $a_{j}$ be the largest integer such that $\left[a_{j}\right] \subseteq D_{j}$. Define

$$
D_{j}^{\prime}=D_{j} \backslash\left[a_{j}\right]
$$

For $1 \leq j_{1}<j_{2} \leq n$, we claim that $D_{j_{1}}^{\prime} \cap D_{j_{2}}^{\prime}=\emptyset$. Suppose to the contrary that $D_{j_{1}}^{\prime} \cap D_{j_{2}}^{\prime} \neq \emptyset$. Assume that $t \in D_{j_{1}}^{\prime} \cap D_{j_{2}}^{\prime}$. Denote $s=a_{j_{1}}+1$. By the definition of $a_{j}$, we see that $s$ is smaller than any integer appearing in $D_{j_{1}}^{\prime}$. Since $t \in D_{j_{1}}^{\prime}$, we have $s<t$. Consider the parts $\alpha_{s}$ and $\alpha_{t}$. Since $D(\alpha)$ is a skyline diagram, it is clear that $D_{j_{1}} \supseteq D_{j_{2}}$, which, along with the fact that $s \notin D_{j_{1}}$, implies $s \notin D_{j_{2}}$. Combining the assumption that $t \in D_{j_{1}} \cap D_{j_{2}}$, we see that $\alpha_{s} \leq \alpha_{t}-2$, and so $\left(\alpha_{s}, \alpha_{t}\right)$ forms a $(0,2)$ patten, leading to a contradiction. This verifies the claim.

Based on the above claim, we can now use the same arguments as in the proof of Theorem 3.2 to conclude the sufficiency. This finishes the proof. 
Acknowledgments. This work was supported by the National Natural Science Foundation of China (Grant No. 11971250) and Sichuan Science and Technology Program (Grant No. 2020YJ0006).

\section{References}

[1] F. Ardila, The Catalan matroid, J. Combin. Theory Ser. A 104 (2003), 49-62.

[2] S. Assaf, Nonsymmetric Macdonald polynomials and a refinement of Kostka-Foulkes polynomials, Trans. Amer. Math. Soc. 370 (2018), 8777-8796.

[3] N. Bergeron and S. Billey, RC-graphs and Schubert polynomials, Experiment. Math. 2 (1993), 257-269.

[4] S. Billey, W. Jockusch and R.P. Stanley, Some combinatorial properties of Schubert polynomials, J. Algebraic Combin. 2 (1993), 345-374.

[5] J. Bonin, A. de Mier and M. Noy, Lattice path matroids: enumerative aspects and Tutte polynomials, J. Combin. Theory Ser. A 104 (2003), 63-94.

[6] P. Brändén and J. Huh, Lorentzian polynomials, Ann. Math., to appear.

[7] M. Demazure, Désingularisation des variétés de Schubert généralisées, Ann. Sci. École Norm. Sup. 7 (1974), 53-88.

[8] M. Demazure, Une nouvelle formule des caractéres, Bull. Sci. Math. 98 (1974), 163-172.

[9] N.J.Y. Fan and P.L. Guo, Vertices of Schubitopes, J. Combin. Theory Ser. A, to appear.

[10] A. Fink, K. Mészáros and A. St. Dizier, Schubert polynomials as integer point transforms of generalized permutahedra, Adv. Math. 332 (2018), 465-475.

[11] A. Fink, K. Mészáros and A. St. Dizier, Zero-one Schubert polynomials, Math. Z., to appear.

[12] S. Gire, Arbres, permutations á motifs exclus et cartes planaire: quelques problémes algorithmiques et combinatoires, Ph.D. Thesis, University of Bordeaux, 1993.

[13] R. Hodges and A. Yong, Multiplicity-free key polynomials, arXiv:2007.09229v1.

[14] J. Huh, J.P. Matherne, K. Mészáros and A. St. Dizier, Logarithmic concavity of Schur and related polynomials, arXiv:1906.09633v3.

[15] B. Ion, Nonsymmetric Macdonald polynomials and Demazure characters, Duke Math. J. 116 (2003), 299-318.

[16] A. Knutson and E. Miller, Gröbner geometry of Schubert polynomials, Ann. Math. 161 (2005), 1245-1318. 
[17] W. Kraśkiewicz and P. Pragacz, Foncteurs de Schubert, C. R. Acad. Sci. Paris Sér. I Math. 304 (1987), 209-211.

[18] W. Kraśkiewicz and P. Pragacz, Schubert functors and Schubert polynomials, European J. Combin. 25 (2004), 1327-1344.

[19] D. Kremer, Permutations with forbidden subsequences and a generalized Schröder number, Discrete Math. 218 (2000), 121-130.

[20] T. Lam, S. Lee and M. Shimozono, Back stable Schubert calculus, arXiv:1806.11233v1.

[21] A. Lascoux and M.-P. Schützenberger, Keys \& standard bases, Invariant Theory and Tableaux (Minneapolis, MN, 1988), 125-144, IMA Vol. Math. Appl., 19, Springer, New York, 1990.

[22] A. Lascoux and M.-P. Schützenberger, Polynômes de Schubert, C. R. Acad. Sci. Paris Sér. I Math. 294 (1982), 447-450.

[23] A. Lascoux and M.-P. Schützenberger, Tableaux and non-commuative Schubert polynomials, Func. Anal. Appl. 23 (1989), 63-64.

[24] P. Magyar, Schubert polynomials and Bott-Samelson varieties, Comment. Math. Helv. 73 (1998), 603-636.

[25] V. Reiner and M. Shimozono, Key polynomials and a flagged Littlewood-Richardson rule, J. Combin. Theory Ser. A 70 (1995), 107-143.

[26] R. Winkel, Diagram rules for the generation of Schubert polynomials, J. Combin. Theory Ser. A. 86 (1999), 14-48. 